\title{
The Application of Laparoscopic B-Ultrasound Microwave Ablation Technology in Liver Metastasis of Colorectal Cancer
}

\author{
Wenfu Zhou, Weimin $\mathrm{Li}^{*}$ \\ Department of Hepatological Surgery, Xiaogan Hospital of Wuhan University of Science and Technology, Xiaogan, China \\ Email: 1600516390@qq.com, *xglwm1962@163.com
}

How to cite this paper: Zhou, W.F. and Li, W.M. (2020) The Application of Laparoscopic B-Ultrasound Microwave Ablation Technology in Liver Metastasis of Colorectal Cancer. International Journal of Clinical Medicine, 11, 62-69.

https://doi.org/10.4236/ijcm.2020.112007

Received: January 17, 2020

Accepted: February 14, 2020

Published: February 17, 2020

Copyright $\odot 2020$ by author(s) and Scientific Research Publishing Inc. This work is licensed under the Creative Commons Attribution International License (CC BY 4.0).

http://creativecommons.org/licenses/by/4.0/

(c) (i) Open Access

\begin{abstract}
Liver is the most common metastasis target organ in the late stage of colorectal cancer. More than $50 \%$ of colorectal cancer patients will have simultaneous or heterochronous liver metastasis. The survival time of patients with colorectal cancer and liver metastasis (CRLM) is short; not all patients can get radical resection of liver metastasis. For this part of patients, microwave ablation technology has been proved to be one of the effective methods for the treatment of liver metastasis. Laparoscopic B-ultrasound ablation also highlights a lot of minimally invasive advantages; this paper reviews the relevant literature of PubMed database, Wanfang database and CNKI database, in order to provide the treatment basis for clinical application of microwave ablation technology under laparoscopic B-ultrasound in the treatment of CRLM. The results showed that the safety and effectiveness of microwave ablation for liver metastases under the location of B-ultrasonic laparoscopy were confirmed, and patients with liver metastases of colorectal cancer who could not be resected could choose this treatment.
\end{abstract}

\section{Keywords}

Laparoscopic Technique, Microwave Ablation, Colorectal Cancer Liver Metastases, Review

\section{Introduction}

Colorectal cancer is one of the most common malignant tumors in China, and liver is the most important target organ for its metastasis. For patients with colorectal cancer and liver metastasis (CRLM), radical resection of liver metastasis 
is still the gold standard, but $80 \%-90 \%$ of patients with liver metastasis have no chance of radical operation. The survival time of patients with liver metastasis was significantly different between the two groups. It is very important to choose the local treatment for CRLM. Thermal ablation is divided into radiofrequency ablation, microwave ablation, laser ablation, high intensity focused ultrasound ablation and cryoablation [1]. Microwave ablation has been proved to be a safe and effective alternative for patients with liver cancer who are not suitable for surgical resection [2]. Some scholars [3] have proved that compared with other non-radical hepatectomy treatments, microwave ablation is a more safe and effective treatment for liver metastasis in CRLM patients. With the development of microwave ablation technology and the development of minimally invasive surgery and the concept of accelerated rehabilitation surgery, microwave ablation technology has been applied in the treatment of CRLM in recent years, and the effect is accurate.

\section{Theoretical Basis of Microwave Ablation under Laparoscope}

1) Principle of microwave ablation under laparoscope

Microwave ablation is one of the local thermal ablation techniques, which uses high frequency electromagnetic wave to produce heat on tissue and make tumor cells produce thermal coagulation. Polar molecules are the material basis of heat generated in living tissue by microwave ablation. The common frequency of microwave ablation for tumor treatment is 915 and $2450 \mathrm{MHz}$. In this microwave field, polar molecules such as water molecules and protein molecules rub with each other, part of kinetic energy is converted into heat energy, so that the tissue is heated. Compared with normal cells, tumor cells have poor heat tolerance. Under the action of high temperature, tumor cells coagulate and necrosis, so as to achieve the purpose of treatment [4]. In order to place the tumor tissue in the microwave field, the surgeon uses the microwave antenna to bury the tumor tissue. Under the B-ultrasonic image, the liver space occupying lesions can be manifested as uneven low, equal, high echo or mixed echo, and there is often complete or incomplete low echo around the tumor, with obvious characteristics. The microwave antenna can be directly buried in the liver lesions under the B-ultrasound location. This treatment scheme has been proved to be safe and effective [5]. In the study of microwave ablation of liver cancer, many scholars [6] have proved that $\leq 5 \mathrm{~cm}$ of liver cancer can achieve therapeutic effect in recent years. The clinical application of laparoscopic technology is now very mature, and the basic hospitals are gradually developing. Most of the elderly and patients with some basic diseases can bear it. With the development of endoscopy technology and microwave ablation technology, the combination of them can expand the treatment of liver metastasis of colorectal cancer.

2) Advantages of microwave ablation under laparoscope

Since the introduction of radiofrequency ablation in 1990, local ablation technology has been gradually applied in the treatment of liver tumors. Com- 
pared with other thermal ablation treatments, microwave ablation has its own advantages. Compared with radio-frequency ablation, it has the characteristics of fast heating, high temperature in tumor, short time, little influenced by carbonized blood flow and no influence by impedance, so it has made great progress in clinical. Some scholars have pointed out that compared with radiofrequency ablation, microwave ablation has lower cost and more advantages for large tumors [7]. In the microwave ablation of liver lesions, it can be divided into percutaneous microwave ablation and laparoscopic microwave ablation, which have received good clinical effect [8] [9]. Compared with the two, laparoscopic microwave ablation can give full play to the advantages of direct vision. It can not only avoid unnecessary side injuries, but also find no lesions that are difficult to be found through auxiliary examination before operation, so that the effectiveness of microwave ablation can be guaranteed [10]. According to related studies, different ablation methods, such as laparotomy, laparoscopy and percutaneous liver tumor ablation, can affect the local recurrence of the tumor. Kuvshinoff et al. [11] have studied that laparoscopic tumor ablation has a lower local tumor recurrence rate than percutaneous puncture. Laparoscopic technology in microwave ablation is not only the role of direct vision or exploration, but also combined with other treatment programs to play its advantages.

\section{The Application of Microwave Ablation under Laparoscope in CRLM}

1) Microwave ablation of B-ultrasonic localization under simple laparoscope

When colorectal cancer patients find liver metastases, they seldom get the opportunity of operation. Effective local treatment is particularly important for CRLM. In clinical application, endoscopic technology has been very mature, most patients can tolerate, laparoscopic direct vision of thermal ablation can try to avoid the side injury of ablation treatment, but also can achieve good ablation effect. With the continuous improvement of minimally invasive technology and the continuous progress of ablation technology, under the common requirements of ensuring effective ablation rate and rapid rehabilitation, the results of laparoscopic B-ultrasound-guided thermal ablation for CRLM are satisfactory. Many scholars [12] [13] have pointed out that it can be used as a local treatment method for non resectable liver metastases. The application of laparoscopic ultrasound system enables surgeons to observe not only the surface of tissues and organs, but also the internal conditions of operation field in real time and from multiple angles. Sun Wenyu et al. [14] showed that for the liver metastases with multiple lesions, microwave ablation was performed on the intrahepatic lesions under the ultrasonic positioning first, and for the lesions with special location invading the hepatic capsule, microwave ablation was performed on the base of the lesions to cut off the blood supply of the tumor completely; the ablation rate was $84.6 \%$ in the reexamination one month after the operation; it was proved that the microwave ablation guided by ultrasound under the laparoscope was not suitable for the unresectable lesions Liver metastasis of colorectal cancer is a safe 
and effective treatment. Luo Hongchang et al. [15] in 2015, 71 cases of HCC with cirrhosis were treated by microwave ablation under the guidance of laparoscopic ultrasound, with good results. Only one patient had serious postoperative complications (1.4\%) and no death. One month later, the reexamination showed that the liver lesions were completely ablated and no residual was found. After a follow-up of $(11.5 \pm 10.1)$ months, local recurrence was found in 4 lesions, distant metastasis in 6 lesions, and local recurrence and distant metastasis in 3 lesions simultaneously. Laparoscopic ultrasound-guided microwave ablation of liver cancer has the advantages of safety, effectiveness, short recovery period and low incidence of complications. Liu Wenbin et al. [16] conducted a comparative analysis of laparoscopic ultrasound-guided and open-ended ultrasound-guided microwave ablation. It was found that patients with laparoscopic treatment had less bleeding, shorter time to recover gastrointestinal function and shorter time to stay in hospital after surgery, while there was no significant difference in operation time, focus ablation time, hospitalization cost and complete ablation rate of focus. Some scholars have proved that the complete ablation rate of tumor is better than that of the percutaneous treatment group, not only minimally invasive but also accurate, and the postoperative recovery of patients is faster [17]. In the study of therapeutic devices, some scholars [18] [19] have made use of pulse microwave and mtDNA targeting single-walled carbon nanotubes as microwave absorbers for the ablation of liver tumors. They have also reached safe and effective conclusions. Ziemlewicz TJ et al. [20] used high-power, air-cooled system and multi-needle combined positioning therapy in clinical application to prove its technical achievements and device safety. Zafar T. et al. [21] proposed that slot antenna combined with microwave analysis is a reasonable choice for microwave ablation, with low cost and simple design; it can analyze the internal temperature distribution curve of malignant tissue, so that the operator can understand the ablation effect. In terms of improving the accuracy of liver metastases under laparoscope, surgeons and ultrasound doctors have also conducted various studies. Yan Shi Yan et al. [22] compared the treatment of real-time contrast-enhanced ultrasound with that of standard ultrasound, and the real-time contrast-enhanced ultrasound under laparoscope is more accurate in positioning liver lesions. There are also some scholars who use MRI [23], PET-MRI [24], robot guided [25] to locate the focus for treatment, but the clinical application is not extensive. Wu Wenbo et al. [26] use the biological simulator to simulate the human body so that the surgeon can carry out surgery practice, which can not only enable the surgeon to understand the operation scheme for surgery simulation, but also shorten the learning curve.

2) Laparoscopic ablation combined with other therapies

a) Combined operation

As the safety and effectiveness of thermal ablation have been gradually confirmed, minimally invasive surgery has gradually matured in clinical practice; laparoscopic ultrasound-guided thermal ablation combined with minimally invasive surgery makes some unresectable liver metastases to be treated, and in 
clinical practice, it is also increasingly favored by the surgeons, retaining more normal liver, so patients benefit. Eng Oliver s et al. [27] conducted a retrospective cohort study. They analyzed the curative effect of laparoscopic radical resection of colorectal cancer combined with radiofrequency ablation in the treatment of liver metastases and that of open radical resection of liver metastases of colorectal cancer. They found that there were statistical differences between the two groups in the comparison of operation time, bleeding volume of the operator, overall postoperative complications and postoperative hospital stay, which also confirmed the laparoscopic operation. The advantages of lower microwave ablation combined with minimally invasive surgery are less complications, faster recovery and better prognosis. Diao Jingfang et al. [28] pointed out that microwave ablation combined with simultaneous operation is safe and effective for liver metastases with diameter $\leq 3 \mathrm{~cm}$ and number $\leq 3$. It is not only the application of thermal ablation in radical resection of colorectal cancer, but also in hepatectomy. Some scholars have proved the effectiveness of microwave ablation combined with hepatectomy in the treatment of HCC and liver metastasis. For the liver lesions with special location or single small lesions, not only enough liver should be reserved, but also the standard of R0 resection should be met. The combination of hepatectomy and microwave ablation has played its advantages [2] [29].

b) Combined with other treatments

Si Zeng Mei et al. [30] used microwave ablation under laparoscope combined with TACE to treat liver cell carcinoma $\geq 5 \mathrm{~cm}$, which proved that it was also safe and effective. Zhao P et al. [31] used TACE combined with microwave ablation to treat primary liver cancer has reliable safety and effectiveness. It is also safe, reliable and easy to operate to use microwave frequency ablation combined with TACE for liver metastases with special location or difficult to remove. Chen Kaiyun et al. [32] used laparoscopic ablation combined with 125I particles to treat liver metastases, and successfully treated the newly found lesions during the operation. The 1-year survival rate and 2-year survival rate of 62 patients were $74.2 \%$ and 59.7\%, respectively. Beerman Marie et al. [33] concluded in 1000 cases that laparoscopic ablation combined with CT imaging computer-aided targeting technology is very successful, and there is no obvious learning curve, and there is less side injury. The research of Yasunori Minami et al. [34] shows that there is a potential effect of heat ablation on immunosuppressive sites of liver metastases, and combined immunotherapy is also expected to become a new direction. The combination of laparoscopic thermal ablation and other treatment schemes has better effect, safety and reliability, and will produce more effective treatment schemes in the future.

\section{Summary and Outlook}

The safety and effectiveness of laparoscopic thermal ablation for primary liver cancer and colorectal cancer with liver metastasis have been gradually con- 
firmed, and it is also one of the effective treatments for colorectal cancer with liver metastasis to achieve R0 resection. Compared with laparotomy and percutaneous ablation, laparoscopic thermal ablation has lower local recurrence rate, less bleeding and shorter hospital stay. The learning curve can span in about 30 cases and is gradually applied in clinical practice. However, most of the conclusions are from retrospective analysis or single center research, which requires more prospective experiments or multi-center clinical experiments, so as to reduce disputes and let patients benefit better. Laparoscopic microwave ablation combined with other treatment schemes also provides a new idea for comprehensive treatment. Multidisciplinary comprehensive diagnosis and treatment is the standard treatment scheme for colorectal cancer patients, and reasonable selection of patients for appropriate treatment is the guarantee of patients' benefits.

\section{Conflicts of Interest}

The authors declare no conflicts of interest regarding the publication of this paper.

\section{References}

[1] Camacho, J.C., Petre, E.N. and Sofocleous, C.T. (2019) Thermal Ablation of Metastatic Colon Cancer to the Liver. Seminars in Interventional Radiology, 36, 310-318. https://doi.org/10.1055/s-0039-1698754

[2] Glassberg, M.B., Ghosh, S., Clymer, J.W., et al. (2019) Microwave Ablation Compared with Hepatic Resection for the Treatment of Hepatocellular Carcinoma and Liver Metastases: A Systematic Review and Meta-Analysis. World Journal of Surgical Oncology, 17, 88. https://doi.org/10.1186/s12957-019-1632-6

[3] Meijerink, M.R., Puijk, R.S., van Tilborg, A.A.J.M., et al. (2018) Radiofrequency and Microwave Ablation Compared to Systemic Chemotherapy and to Partial Hepatectomy in the Treatment of Colorectal Liver Metastases: A Systematic Review and Meta-Analysis. Cardio Vascular and Interventional Radiology, 41, 1189-1204. https://doi.org/10.1007/s00270-018-1959-3

[4] Yu, J. and Liang, P. (2017) Status and Advancement of Microwave Ablation in China. International Journal of Hyperthermia, 33, 278-287. https://doi.org/10.1080/02656736.2016.1243261

[5] Glassberg, M.B., Ghosh, S., Clymer, J.W., et al. (2019) Microwave Ablation Compared with Radiofrequency Ablation for Treatment of Hepatocellular Carcinoma and Liver Metastases: A Systematic Review and Meta-Analysis. OncoTargets and Therapy, 12, 6407-6438. https://doi.org/10.2147/OTT.S204340

[6] Zhang, T.T., Li, K.Y., Luo, H.C., et al. (2014) Long-Term Outcomes of Percutaneous Microwave Ablation versus Repeat Hepatectomy for Treatment of Late Recurrent Small Hepatocellular Carcinoma: A Retrospective Study. Chinese Medical Journal, 94, 2570-2572.

[7] Zhang, X.G., Zhang, Z.L., Hu, S.Y., et al. (2014) Ultrasound-Guided Ablative Therapy for Hepatic Malignancies: A Comparison of the Therapeutic Effects of Microwave and Radiofrequency Ablation. Acta Chirurgica Belgica, 114, 40-45.

https://doi.org/10.1080/00015458.2014.11680975 
[8] Wang, J.B., Liang, P., Yu, J., et al. (2014) Clinical Outcome of Ultrasound-Guided Percutaneous Microwave Ablation on Colorectal Liver Metastases. Oncology Letters, 8, 323-326. https://doi.org/10.3892/ol.2014.2106

[9] Salvatore, G., Duilio, P., Alessandro, T., et al. (2016) Laparoscopic Approach for Thermoablation Microwave in the Treatment of Hepatocellular Carcinoma: A Single Center Experience. Journal of Laparoendoscopic and Advanced Surgical Techniques, 26, 808-811. https://doi.org/10.1089/lap.2016.0373

[10] Umberto, C., Giulia, N., Alessandro, V., et al. (2014) Laparoscopic Microwave Ablation in Patients with Hepatocellular Carcinoma: A Prospective Cohort Study. HPB (Oxford), 16, 979-986. https://doi.org/10.1111/hpb.12264

[11] Kuvshinoff, B.W. and Ota, D.M. (2002) Radiofrequency Ablation of Liver Tumors: Influence of Technique and Tumor Size. Surgery, 132, 605-611.

https://doi.org/10.1067/msy.2002.127545

[12] Vogl, T.J., Farshid, P., Naguib, N.N.N., et al. (2014) Thermal Ablation of Liver Metastases from Colorectal Cancer: Radiofrequency, Microwave and Laser Ablation Therapies. La Radiologia Medica, 119, 451-461. https://doi.org/10.1007/s11547-014-0415-y

[13] Li, W.D., Zhou, X., Huang, Z.J., et al. (2017) Short-Term and Long-Term Outcomes of Laparoscopic Hepatectomy, Microwave Ablation, and Open Hepatectomy for Small Hepatocellular Carcinoma: A 5-Year Experience in a Single Center. Hepatology Research, 47, 650-657. https://doi.org/10.1111/hepr.12785

[14] Sun, W.-Y., Jiang, H.-L., Yu, H., et al. (2012) Ultrasound Guided Combined with Laparoscopic Microwave Ablation in Treatment for Special Site Liver Cancer. Chinese Journal of Bases and Clinics in General Surgery, 19, 188-191.

[15] Luo, H.C., Zhang, T.T., Zhang, W., et al. (2015) Laparoscopic Microwave Ablation Guided by Laparoscopic Ultrasound in Treating Hepatic Cellular Carcinoma with Liver Cirrhosis. Journal of Clinical Ultrasound in Medicine, 17, 173-176.

[16] Liu, W.B., Ma, J.L., Jia, W.D., et al. (2019) Comparison of Laparoscopic Microwave Ablation and Open-Abdominal Microwave Ablation for Primary Hepatic Carcinoma under Ultrasonic Guidance. Journal of Hepatobiliary Surgery, 27, 249-253.

[17] He, L.-G., Che, S.-Y., Chen, Q.-S., et al. (2018) Comparison of Clinical Effects of Percutaneous Microwave Ablation and Laparoscopic Microwave Ablation in Treatment of Small Hepatocellular Carcinoma Patients with Special Site. Chinese Journal of Liver Diseases (Electronic Version), 10, 74-77.

[18] Bedoya, M., del Rio, A.M., Chiang, J., et al. (2014) Microwave Ablation Energy Delivery: Influence of Power Pulsing on Ablation Results in an ex Vivo and in Vivo Liver Model. Medical Physics, 41, Article ID: 123301. https://doi.org/10.1118/1.4901312

[19] Wen, L.W., Ding, W.Z., Yang, S.H., et al. (2016) Microwave Pumped High-Efficient Thermoacoustic Tumor Therapy with Single Wall Carbon Nanotubes. Biomaterials, 75, 163-173. https://doi.org/10.1016/j.biomaterials.2015.10.028

[20] Ziemlewicz, T.J., Hinshaw, J.L., Lubner, M.G., et al. (2015) Percutaneous Microwave Ablation of Hepatocellular Carcinoma with a Gas-Cooled System: Initial Clinical Results with 107 Tumors. Journal of Vascular and Interventional Radiology, 26, 62-68. https://doi.org/10.1016/j.jvir.2014.09.012

[21] Zafar, T., Zafar, J. and Zafar, H. (2014) Development and Microwave Analysis of Slot Antennas for Localized Hyperthermia Treatment of Hepatocellular Liver Tumor. Australasian Physical and Engineering Science in Medicine, 37, 673-679.

https://doi.org/10.1007/s13246-014-0300-y 
[22] Yan, S.-Y., Zhang, Y., Sun, C., et al. (2016) Comparison of Real-Time Contrast-Enhanced Ultrasonography and Standard Ultrasonography in Liver Cancer Microwave Ablation. Experimental and Therapeutic Medicine, 12, 1345-1348. https://doi.org/10.3892/etm.2016.3448

[23] Murakami, K., Naka, S., Shiomi, H., et al. (2015) Initial Experiences with MR Image-Guided Laparoscopic Microwave Coagulation Therapy for Hepatic Tumors. Surgery Today, 45, 1173-1178. https://doi.org/10.1007/s00595-014-1042-x

[24] Nielsen, K., Scheffer, H.J., Pieters, I.C., et al. (2014) The Use of PET-MRI in the Follow-Up after Radiofrequency and Microwave Ablation of Colorectal Liver Metastases. BMC Medical Imaging, 14, Article No. 27. https://doi.org/10.1186/1471-2342-14-27

[25] Beyer, L.P., Pregler, B., Niessen, C., et al. (2016) Robot-Assisted Microwave Thermoablation of Liver Tumors: A Single-Center Experience. International Journal of Computer Assisted Radiology and Surgery, 11, 253-259. https://doi.org/10.1007/s11548-015-1286-y

[26] Wu, W.B., Xue, Y.F., Wang, D., et al. (2014) A Simulator for Percutaneous Hepatic Microwave Thermal Ablation under Ultrasound Guidance. International Journal of Hyperthermia, 30, 429-437. https://doi.org/10.3109/02656736.2014.957738

[27] Eng, O.S., Tsang, A.T., Moore, D., et al. (2015) Outcomes of Microwave Ablation for Colorectal Cancer Liver Metastases: A Single Center Experience. Journal of Surgical Oncology, 111, 410-413. https://doi.org/10.1002/jso.23849

[28] Diao, J.F., Mo, J.Q., Ye, Q., et al. (2017) Clinical Efficacy of Laparoscopic Ultrasound-Guided Microwave Ablation for Concurrent Treatment of Colorectal Liver Metastasis. Chinese Journal of Hepatic Surgery, 6, 312-315.

[29] Han, J.-B., Kong, F.-W., Ding, H., et al. (2017) Hepatectomy Combined with Microwave Ablation of the Spleen for Treatment of Hepatocellular Carcinoma Complicated with Splenomegaly: A Retrospective Study. Molecular and Clinical Oncology, 6, 204-208. https://doi.org/10.3892/mco.2016.1111

[30] Si, Z.-M., Wang, G.-Z., Qian, S., et al. (2016) Combination Therapies in the Management of Large $(\geq 5 \mathrm{~cm}$ ) Hepatocellular Carcinoma: Microwave Ablation Immediately Followed by Transarterial Chemoembolization. Journal of Vascular and Interventional Radiology, 27, 1577-1583. https://doi.org/10.1016/j.jvir.2016.02.014

[31] Zhao, P., Zheng, J.S., Zhang, H.H., et al. (2016) Efficacy Evaluation and Exploration of TACE Combined with CT-Guided Precision Microwave Ablation Treatment for Primary Liver Cancer. Chinese Journal of Oncology, 38, 138-145.

[32] Chen, K.-Y., Xiang, G.-A., Wang, H.-N., et al. (2007) Combination of Laparoscopic Excision, Iodine-125 and Radiofrequency Ablation in the Treatment of Hepatic Metastasis. Chinese Journal of Digestive Endoscopy, 24, 335-337.

[33] Beermann, M., Lindeberg, J., Engstrand, J., et al. (2019) 1000 Consecutive Ablation Sessions in the Era of Computer Assisted Image Guidance-Lessons Learned. European Journal of Radiology Open, 6, 1-8. https://doi.org/10.1016/j.ejro.2018.11.002

[34] Yasunori, M., Naoshi, N. and Masatoshi, K. (2019) Radiofrequency Ablation of Liver Metastasis: Potential Impact on Immune Checkpoint Inhibitor Therapy. European Radiology, 29, 5045-5051. https://doi.org/10.1007/s00330-019-06189-6 